\title{
Clinical Decision Support Systems
}

\author{
Dejan Dinevski, Uroš Bele, Tomislav Šarenac, \\ Uroš Rajkovič and Olga Šušteršič \\ Faculty of Medicine, University of Maribor, University Clinical Center Maribor \\ Faculty of Organizational Sciences, University of Maribor and \\ Faculty of Health Sciences, University of Ljubljana \\ Slovenia
}

\section{Introduction}

In the chapter we are presenting the theoretical background, state of the art and modern research trends of Clinical decision support systems (CDSS). The challenges for success are derived and our experience is described with the presentation of a good practice example of employing CDSS in telemedicine.

CDSS systems are increasingly often integrated into telemedicine clinical practice. In addition to using the same resources, namely digitally coded clinical data, CDSS systems are able to enhance the quality of telemedicine services in many cases.

CDSS are computer applications that are designed to help health-care professionals with making clinical decisions about individual patients (Shortliffe and Cimino 2006; Berner 2007). In other words, CDSS are active knowledge systems, which use two or more items of patient data to generate case-specific advice (Wyatt and Spiegelhalter 1991).

These kinds of software use relevant knowledge, rules within a knowledge base and relevant patient and clinical data to improve clinical decision making on topics like preventive, acute and chronic care, diagnostics, specific test ordering, prescribing practices (National Electronic Decision Support 2003; Pearson, Moxey et al. 2009). A CDSS correlates data about patient traits with a trustworthy knowledge base to guide a clinician with patient-specific advice, assessments or recommendations. Clinicians, health-care staff or patients can manually enter patient characteristics into the computer systems; alternatively, electronic medical records (EMR) can be queried for retrieval of patient characteristics. These kinds of decision-support systems allow the clinicians to spot and choose the most appropriate treatment. Provided decision-support is based on processes of sophisticated outcomes assessment and algorithms that use knowledge bases to inquire after the newest developments about best practice (Remmlinger 2002; Garg, Adhikari et al. 2005).

Regardless of how we choose to define CDSS, we have to accept that the field of CDSS is rapidly advancing and unregulated. It has a potential for harm, if systems are poorly designed and inadequately evaluated, as well as a huge potential to benefit, especially in health care provider performance, quality of care and patient outcomes (Delaney, Fitzmaurice et al. 1999; Pearson, Moxey et al. 2009). 


\section{Clinical decision-making}

There are some principal categories to take into account while striving for excellent decisionmaking: a) accurate data, b) applicable knowledge, c) appropriate problem-solving skills (Shortliffe and Cimino 2006).

Patient data must be adequate to make a valid decision. The problem arises, when the clinician is met with an overwhelming amount of specific and unspecific data, which he/she cannot satisfactorily process. Therefore it is important to assess when additional facts will confuse rather than clarify the patient's case. For example, usual setting for such a problem are intensive-care units, where practitioners must absorb large amounts of data from various monitors, be aware of the clinical status, patient history, accompanying chronic illnesses, patient's medication and adverse drug interactions, etc. - and on top of that make an appropriate decision about the course of action. The quality of available data is of equal importance. Measuring instruments and monitors should be as accurate as technologically possible, since erroneous data could have serious adverse effect on patient-care decisions.

Knowledge used in decision-making process must be accurate and current. It is of major importance that the deciding clinician has a broad spectrum of medical knowledge and access to information resources, where it is possible to constantly revise and validate that knowledge. For a patient to receive appropriate care, the clinician must be aware of the latest evidence-based guidelines and developments in the area of the case in question. It is in clinician's hands to bring proven therapies from research papers to the bedside. CDSS analogously needs an extensive, well-structured and current source of knowledge to appropriately serve the clinician.

Above all, good problem-solving skills are needed to utilize available data and knowledge. Deciding clinicians must set appropriate goals for each task, know how to reason about each goal and take in to account the trade-offs between costs and benefits of therapy and diagnostics. In further reflection, we should not neglect, that skilled clinicians draw extensively from their personal experience.

By incorporating patient-specific data and evidence-based guidelines or applicable knowledge base, the CDSS can improve quality of care with enhancing the clinical decisionmaking process (General Practice Electronic Decision Support 2000).

In order to be able to construct applicable CDSS, it is imperative to have a broader-based understanding of medical decision-making as it occurs in the natural setting. Designing CDSSs without understanding the cognitive processes underlying medical reasoning and decision analysis is pliable for ineffectiveness and failure for implementation into everyday clinical workflow (Patel, Kaufman et al. 2002).

\subsection{Clinical decision analysis (problem solving)}

Decision analysis is a quantitative evaluation of the outcomes that result from a set of choices in a specific clinical situation and is the methodology usually chosen for clinical problem solving. This process is often implicit and occurs within internal algorithms and heuristics (mental shortcuts) that the clinician has developed and acquired over time. Decision analysis, by requiring a specific model structure and assessment of various likelihoods and values of the outcomes, makes the decision process explicit and more inclined to examination, discussion, and re-validation. Decision models are often used as analytic tools to conduct cost-effectiveness analyses, since this methodology can be used to find the expected value of predicted outcomes. 
The range of clinical problems appropriate for decision analysis is vast. Such problems focus upon a specific decision, where there is a tradeoff involved, which means that one of the choices considered is not necessarily unambiguously superior. As an example, a diagnostic test carries some risk, but it trades off with more appropriate therapy and better patient outcome when treatment is directed by the results of that test. Decision-making is rarely a clear-cut process - it therefore inevitably involves compromises.

In general, decision analyses have been developed to:

- Assist in clinical decision-making for a specific individual patient;

- Estimate optimal strategies for classes of patients with specific clinical characteristics in given situations;

- Link estimates of both clinical and economic outcomes (cost-effectiveness analysis) to help in forming issues regarding health policy;

- Provide estimates of expected outcomes in situations where classical methods such as randomized trials are either impossible or impractical.

There are several advantages of using decision analysis to investigate options involving a single patient. Placing the problem in an explicit, analytic context forces clinicians to make their assumptions clear. Decision analyses can directly incorporate issues regarding quality of life and how the particular patient values various outcomes. An example of such a decision analysis is weighing the risks and benefits of prophylactic mastectomy (breast removal) and oophorectomy (removal of ovaries) for patients with BRCA1 or BRCA2 mutations. One has to combine several kinds of data - estimates of the increased risks of ovarian and breast cancer for women with the mutations, general cancer incidence and last but not least, the risks that the surgical procedures impose. The analysis in a certain point of time demonstrates, using the best currently available data, that life expectancy for women who carry such mutations was increased by as much as 5.3 years with prophylactic mastectomy and by as much as 1.7 years with prophylactic oophorectomy.

Developing and constructing a decision analysis follows a logical series of steps (problemsolving). Problems or errors in any step can alter the eventual results; thus, proper adherence to each of these steps is important both from the view of a researcher conducting an analysis and a clinician interpreting the results. The steps are (Roberts 2011):

- Frame the question - The process of defining the scope and boundaries of the particular clinical situation to be analyzed. In other words at this stage one is to analyze the new data which suggest a problem and identify the problem as it appears.

- Structure the clinical problem - Structuring the problem simply means constructing a decision model that represents the relevant components of the problem. We need to think through what the alternative solutions might be and create a list of alternatives hypotheses. In the clinical setting these might be potential therapeutic options for a set diagnosis. The mathematical representation of a decision model is called a decision tree and is composed of several different elements. Elements combined into trees contain an amount of detail arbitrarily designated. There is a permanent tension between increasing the level of detail to be as clinically realistic as possible versus the feasibility of model construction, validation, and presentation. The more detail desired, the more difficult the model is to construct, validate, and present. However, the less detailed the analysis and the greater the number of simplifying assumptions, the more vulnerable is the analysis to attack for lacking clinically meaningful elements.

- Estimate the relevant probabilities - Once a decision tree has been structured, the numeric values of various probabilities need to be determined. There are many sources 
of data that can be used to make these determinations. Although there is a generally accepted hierarchy of study quality (randomized controlled trial $>$ cohort study $>$ administrative databases $>$ systematic reviews), such ratings are not always useful for decision analysis since the particular study type may not be conducive in estimating a given parameter. As an example, a randomized controlled trial is an excellent source for comparing one therapy versus another, but it is a poor source of data regarding the incidence of a particular disease. Thus, it is important to tailor the data source to the type of data required.

However there might not be enough evidence to assign relevant probabilities to candidate solutions or even to structure the clinical problem and find suitable alternative solutions in the first place (see previous step). Thus the process is bound to reform with a new search to fill the present "knowledge gap". Potentially many iterations of this cycle of data gathering and hypotheses generation may occur until the decision-making clinician has enough clarity to move on through the process. Clinical decision-analysis is hence a fundamental fuel for further research, evidence collection and problem re-evaluation - all for the purpose of finding suitable solutions that will help resolving the clinical situation. CDSS can be programmed in a way that they rearrange themselves for a specific problem, search for relevant evidence and even create new knowledge (Coiera 2003).

- Estimate the values of the outcomes - Different solutions to a problem have different outcomes. The structure of the problem defines the specific outcome measure to be used. For example, if death was a possible outcome of one or more strategies, life expectancy would be an appropriate outcome measure. The most important aspect in assigning outcomes is that they are measured in the same units across all branches. A useful feature of decision analysis is that a given model can be evaluated using different outcome measures. As an example, in addition to survival, the clinician may want to track the effect of different therapies upon the rate of stroke, myocardial infarction, pulmonary embolization, etc., across various treatment options. The decision model can be analyzed using any of those outcomes.

- Analyze the tree - The preferred type of analysis of a problem utilizes the strategy that maximizes the expected value of the outcome. As an example, if the outcome of interest was life expectancy, the result of a decision analysis would be of the form: "the average life expectancy with strategy A is 11.3 years versus 8.6 years with strategy B; therefore strategy A is the optimal strategy." Such a tree might represent the choice between two therapies, where one is preferred in terms of life expectancy.

- Test the model's assumptions - The results obtained from a decision analysis depend upon the accuracy of the data used to estimate the probabilities and outcomes. It is rarely the case that estimates are known with complete certainty. One of the major advantages of decision analysis models is their ability to rapidly test their assumptions and input data - to validate the decision model by performing a sensitivity analysis. The model's answer can be compared with the "true" answer to validate the model structure by evaluating a tree using parameters for which the result is known a priori. As an example, in a choice between a more effective (but riskier) surgical therapy and a less effective (but safer) medical therapy, a sensitivity analysis that postulated a zero mortality rate for the surgical intervention should advocate the surgical arm since there would be no downside risk. 
- Interpret the results - Interpreting the results of a decision analysis is often the most complicated task. The ability of a decision analysis to explore how the optimal strategy in a particular clinical situation changes with variation in assumptions (and therefore to identify areas for further data needs) is often one of the most powerful attributes of this type of analysis. Several details of an analysis should be examined prior to using the results to change practice: a) The patient population should closely match the patients seen by the clinician. b) The reader needs to assess the strength of the result (sensitivity analysis). If sensitivity analyses indicate that the optimal choice is strongly dependent upon a given parameter, one should try to develop accurate measures of the parameter estimate.

Reasoning in a medical context involves uncertainty to a high extent, combined with constrained resources it therefore leads to increased use of heuristic strategies. Greatest advantage of heuristics is in limiting the extent of purposeful search through data sets and knowledge bases. By reducing redundancy, heuristics have substantial practical value. A significant part of a clinician's cognitive effort is based on heuristic thinking, the reasoning is inductive with assigned probability for each possible choice. However, the use of heuristics introduces considerable bias in medical reasoning, often resulting in a number of conceptual and procedural errors. These include misconceptions about laws that govern probability, false understanding of manifestation of general rules to a specific patient at the point of care, neglect and false validation of prior probabilities and therapeutic actions. Above all, the cognitive biases that unavoidably affect a human-decision maker are often the hallmark of decision uncertainty (Holyoak 2005).

If the designers of CDSSs are to create effective systems which would be successfully implemented into the workflow, providing the clinician with the necessary support of their decision-making abilities, they must be ready to accept the general paths of clinical decisionmaking process, described above. Efficiently designed and correctly implemented CDSS can elicit extensive positive externalities to the health-workers' performance and improve patient outcome thus affect the gross quality of healthcare.

\section{Structure of clinical decision support systems}

CDSSs vary very much in their design. The basic principles of structure and design had also changed substantially in the last decade. Various traits of CDSSs are related to or have a direct effect on clinical effectiveness, functionality, error prevention, potential for acceptance in the clinical world, system portability, cost-effectiveness etc. It is thus important to characterize CDSSs in a way to best understand the diversity of CDSS. The knowing of classification, conjunct with an idea of general clinical decision-making processes described above, present a powerful set of basic facts that are useful for CDSSs designers and evaluators.

In this chapter, we will try to characterize CDSS, combining several resources to create a comprehensive classification that capture key elements of CDSS design and function (Coiera 2003; Sim and Berlin 2003; Shortliffe and Cimino 2006; Berner 2007; Greenes 2007). According to Sim and Berlin 2003, the CDSS can be categorized along 5 axes.

\subsection{Context axes}

1. Clinical setting.

- Inpatient setting

- Outpatient setting 


\section{Clinical Task.}

- Diagnostic assistance.

Based on the patient's data and the system's knowledge base, the CDSS provides likely diagnoses. Diagnostic assistance can be coupled with complex data-retrieval systems, like ECG. It seeks to identify "what is true" for a specific patient.

- Therapy critiquing and consulting.

This function can for example be incorporated into clinician's order-entry. It assesses the therapy, looks for inconsistencies, errors, cross-references for drug interactions and prevents prescribing of allergenic drugs. It has been shown that the necessity of a clinician to provide a statement of an appropriate reason, if he is or she is not to follow the recommendations, significantly increases the clinical importance of CDSSs. The CDSS can use protocols and evidence-based guidelines, combined with patient's facts, acquired from EMR, to provide an optimal treatment plan and help following it. These kinds of CDSSs address the question "what to do" with a patient and are often combined with recommendations about further diagnostic processing (i.e. which tests to order, X-ray, CT etc.). Such software can generate additional questions as to provide even more specific advice about further therapy (and diagnosis).

- Drug dosing or prescribing.

CDSS have the power to reduce toxic drug levels, reduce medical errors, change prescribing in accordance to guideline recommendations and reduce time to achieving therapeutic control. If connected to EMR, the system can prevent prescription of drugs that cause allergic reactions. Such systems have been widely accepted, since they are well integrated into a routine part of the clinician's workflow and they provide automated order-entry forms and electronic transmission to pharmacies. Overall prescription of medication is one of the commonest tasks of a physician and also one of the commonest clinical tasks where CDSSs arebeing applied.

- $\quad$ Test selection.

- Alerts and reminders.

An expert system that is integrated into a monitoring device or health-care information system (e.g. laboratory information system, EMR) can provide realtime sound, visual or tactile alerts thru various communication tools (e.g. e-mail, SMS, pager). Reminder systems are designed to remind the clinician of crucial tasks that need to be done before a certain event (e.g. fasting before endoscopy, no anticoagulants before abdominal surgery).

- Information retrieval.

Relevant information retrieval through world wide web or comprehensive knowledge bases.

- Image recognition and interpretation.

Clinical images from CT, MRI, angiograms etc. can nowadays be partially automatically interpreted. More importantly CDSS can function as a mass screening tool, where software flags critical images, which require clinician's special attention.

- Prevention.

- Screening. 
- $\quad$ Expert laboratory system.

- Chronic disease management.

\subsection{Knowledge axes}

This axes deals with the sources, quality and customization of the CDSS's knowledge and data.

1. Clinical knowledge source.

It can be derived from high-quality sources (e.g. randomized-controlled trials, systematic reviews, national or professional society guidelines) and/or from participation of clinicians that will eventually use the system.

2. Data source.

The patient-specific data can be retrieved from computerized order entry, medical instrument (e.g. blood pressure measuring device), EMR or other data repository. The data may also be gathered from a paper chart or a person. In that case data must be entered in the system using a data input intermediary (see bellow). This characteristic affects the likelihood of CDSS adoption in practice to a high extent. It has been shown, that automatic computerized provision of data to the system (e.g. from EMR) is preferred.

3. Data source intermediary is a clinician who inputs data into data source (see above). Intermediaries could also be patients themselves.

\section{Data coding.}

For various reasons (e.g. funding, epidemiology) it is desirable to use a widely use coding schema, i.e. ICD-10, SNOMED. Obviously the data could also be in plain text.

\section{Data customization.}

The more CDSS produces patient-specific targeted recommendations, customized to age, gender, concomitant diagnoses etc., more it has a probability of clinical relevance and effectiveness.

6. Update mechanism.

As stated above, knowledge base ought to be current and constantly up-to-date.

We can divide CDSS to knowledge based and non-knowledge based systems.

The knowledge-based systems (a.k.a. expert systems) mostly consist of three parts - the knowledge base, inference engine and the mechanism to communicate. They contain expert clinical knowledge about very specific facts and tasks and are able to reason with the input of data from all the various sources stated above. These systems usually use knowledge in form of IF-THEN rules and probabilistic associations between compiled data. The inference engine lies at the core of the artificial intelligence part of knowledge-based systems - it combines and correlates knowledge base rules with the patient's data. Basically the inference engine reasons with the given information to form new conclusions (Wikipedia 2011).

The non-knowledge-based systems rather use principle of machine learning in the form of i.e. neural networks or genetic algorithms, where computers learn from past experience and/or find patterns in clinical data of an individual.

\subsection{Decision support axes}

Addressing a suitable decision-making process is probably the most important dimension of CDSS.

1. Reasoning method. Some of CDSS reasoning engines are: 
- Rule-based systems. A rule-based system uses different expert knowledge bases in form of expressions that can be evaluated as IF-THEN rules (production rules). Such a system is an example of heuristic approach in which individual logical statements in the form of production rules are obtained by observing human experts, or interviewing and debriefing them, and then combined in an attempt to emulate the reasoning processes of experts. This approach was first used in the MYCIN (Shortliffe 1976), with the goal of choosing appropriate antimicrobial therapy for a patient.

- Neural networks. Artificial neural network is a non-knowledge-based adaptive CDSS that uses machine learning to learn from experiences and recognize patterns in clinical information.

- Bayesian network. A typical knowledge-based decision-making system is the Bayesian network (a.k.a belief network or causal probabilistic network) that shows probabilistic relationships between sets of variables - diseases and symptoms, based on conditional probability according to Bayes theorem. It is a network with a explicit requirement, that the relationships be causal. Such a network helps to model the progression of a disease over time and the interaction between diseases; a big road-block however is that medical knowledge sometimes finds it difficult to directly specify what is the effect and what the cause.

- Model based systems. The latest achievement is patient specific modeling (see section 5.2.).

- Logical condition. Logical reasoning makes decisions according to the value of a given variable. The results of a decision-making process are different if the value is within or outside of the set boundaries.

- Data mining and machine learning. These methods are based on probabilistic decision-making according to the system's database. The ideal databases should be large and well constructed, so that they allow precise retrieval of patients similar to a current patient. Analysis of responses of those patients to various treatments is used to decide upon the best treatment for the current patient.

- Genetic algorhytm. As a non-knowledge-based method it uses iterative processes to rearrange itself and provide an optimal solution based on the patient data.

\section{Clinical urgency.}

Provision of decision support to decisions that have to be made urgently. CDSS should first provide its function to issues with clear clinical priority, according to the principle "treat first what kills first". This characteristic leads to better patient outcomes and physicians performance.

3. Recommendation explicitness.

Users are more likely to follow explicit recommendations, providing concrete course of action.

4. Response requirement.

The using clinician may be required to provide justification for the way he/she responded to the recommendation provided by the CDSS. This could be done in a form of acknowledgement of recommendation, with a statement of what alternative action was taken and with an explanation for non-compliance. 


\subsection{Information delivery axes}

This axes deals with the delivery of newly produced information to the user.

1. Delivery format.

Paper-based, online (via internet or integrated into EMR), via other technology - phone, pager, e-mail etc.

2. Delivery mode.

The recommendations can be delivered after the decision-maker has requested so or on the other hand it could be delivered without the consent in form of an alert, reminder or optimization request. In the first case the clinician has to make an additional effort, recognize when the advice would be useful, "go to the program" and enter data to request diagnostic or therapeutic assessment, thus the program is passive.The so-called "push systems" that automatically provide recommendations may be more effective and substantially more used. They play an active role with providing decision support as a byproduct of data-managing activities (e.g. monitoring, EMR supervision). System decision logic is in a way integrated within a patient's database that is already being gathered from various sources and provides results of its decision analysis without an additional effort of the clinician. A valid point to consider here is how to avoid so-called "alarm fatigue", where the clinician is over-warned to minor discrepancies which are otherwise noted and commonly understood.

3. Action integration.

It is imperative, that the CDSS provides the ability to the decision-maker to exert the recommended actions with ease. For example, the software can, while providing prompts on therapy-critique, also provide direct links to order-entry forms and therapy planning section of EMR. The action of therapy change should be completed within a range of few clicks, for example with checking a mark. Action integration unambiguously adds to wider acceptance and usability of CDSS.

4. Explanation availability.

It is a function, where the system provides an explanation of its recommendations, through e.g. links to evidence-based articles, books or directly from the knowledge base.

\subsection{Workflow axes}

CDSS can be seen as a process, however at the point of care it is virtually an intervention of technology, that could also act as an interruption. Systems that are synergistic with the institution's workflow are likely to experience higher usage and prove to be more effective in optimizing practitioner's performance.

\section{Success factors of CDSS}

Despite the fact, that the computerized CDSSs were continuously in development since the 1970s, their impact on routine clinical practice has not been as strong as expected. The potential benefits of using electronic decision support systems in clinical practice fall into three broad categories (Coiera 2003):

1. Improved patient safety (reduced medication errors and unwanted adverse events, refined ordering of medication and tests);

2. Improved quality of care (increasing clinicians' time allocated directly to patient care, increased application of clinical pathways and guidelines, accelerate and encourage the use of latest clinical findings, improved clinical documentation and patient satisfaction); 
3. Improved efficiency of health-care (reducing costs through faster order processing, reductions in test duplication, decreased adverse events, and changed patterns of drug prescribing, favoring cheaper but equally effective generic brands).

Developing CDSSs is a challenging process, which may lead to a failure despite our theoretical knowledge about the topic. Understanding the underlying causes, which lead either to success or either to failure, may help to improve the efficiency of CDSS development and deployment in day-to-day practice. Failures can originate from various developmental and implementation phases: failure to technically complete an appropriate system, failure to get the system accepted by the users and failure to integrate the system in the organizational or user environment (Brender, Ammenwerth et al. 2006).

There is an estimation that $45 \%$ of computerized medical information systems fail because of user resistance, even though these systems are technologically coherent. Some reasons for such a high percentage of failure may derive from insufficient computer ability, diminished professional autonomy, lack of awareness of long-term benefits of CDSS-use and lack of desire to change the daily workflow (Zheng, Padman et al. 2005). There is also clear evidence that CDSS services are not always used when available, since too numerous systems' alerts are being overridden or ignored by physicians (Moxey, Robertson et al. 2010).

Despite the problems and failures that might accompany CDSSs, these systems have still been proven to improve drug selection and dosing suggestions, reduce serious medication errors by flagging potential drug reactions, drug allergies and identifying duplication of therapy, they enhance the delivery of preventive care services and improve adherence to recommended care standards.

Recent studies suggest that there are some CDSS features crucial to success of these systems (Kawamoto, Houlihan et al. 2005; Shortliffe and Cimino 2006; Pearson, Moxey et al. 2009; Moxey, Robertson et al. 2010):

- CDSS should provide decision support automatically as part of clinicians' workflow, since systems where clinicians were required to seek out advice manually have not been proven as successful.

- Decision support should be delivered at the time and location of decision-making. If the clinician has to interrupt the normal pattern of patient care to move to a separate workstation or to follow complex, time-consuming startup procedures it is not likely that such system will be good accepted.

- Systems that were provided as an integrated component of charting or ordering systems were significantly more likely to succeed than alone standing systems. Generally speaking, the decision-support element should be incorporated into a larger computer system that is already part of the users' professional routine, thus making decision support a byproduct of practitioners' ordinary work practices.

- Computerized systems have been reported to be advantageous over paper-based systems.

- Systems should provide recommendation rather than just state a patient assessment. For instance, system recommends that the clinician prescribes diuretics for a patient rather just identifying patient being cardiologically decompensated.

- CDSS should request the clinician to record a reason for not following the systems' advice (the clinician is asked to justify the decision with a reason, e.g. "The patient refused").

- It should promote clinicians' action rather than inaction. 
- No need for additional clinical data entry. Due to clinicians' effort required for entering new patient data, they tend to avoid this process, which is essential for new decision support. Systems should rather acquire new data automatically (e.g. data retrieval from EMR).

- The system should be easy to navigate and use, e.g. with quick access and minimal mouse clicks for desired information.

- Timing and frequency of prompts are of great importance. For instance if there are too many messages, this might only lead to ignoring all of them and consequently to missing important information. The timing is as well of great importance - the alerts shouldn't appear at inappropriate times and interrupt the workflow.

- The presentation of data or information on CDSSs shouldn't be too dense or the text to small. Researchers also suggest the use of blinking icons for important tasks or the arrangement of interactions according to their urgency.

- Decision support results should be provided to both clinicians and patients. Studies have shown beneficial effect of such actions, because they stimulate the clinicians to discuss treatment options with patients, and consequently make the latter feel more involved in their medical treatment.

- Periodic feedback about clinician's compliance with system decision-making.

What these features have in common is that they all make it easier for clinicians to implement the CDSS into their workflow, thus making it easier to use. An effective CDSS must minimize the effort to receive and act on system recommendations. Clinicians found it also very practical if the CDSS would back up its decision-making with linking it to other knowledge resources across the intranet or Internet. In their opinion the safety and drug interaction alerts were the most helpful feature. Above all the organizational factors, such as computer availability at the point of care and technical perfection of CDSS hardware and software are crucial to implementation (Moxey, Robertson et al. 2010).

Kawamoto 2005 suggests that the effectiveness of CDSS remains mainly unchanged when system recommendations are stated more strongly and when the evidence supporting these prompts is expanded and includes institution-specific data. Similarly, the effectiveness and functionality remains unaltered when recommendations are made more specific. Interestingly the CDSSs didn't achieve wanted results when local clinicians were recruited into the system development process nor when bibliographic citations were provided to support the system made recommendations (Kawamoto, Houlihan et al. 2005).

To sum up, when developing CDSSs, there are factors beyond software and content that must be taken into consideration. Fundamental issues include availability and accessibility of hardware, sufficient technical support and training in use of the system, the level of system integration into clinical workflow and the relevance and timeliness of the clinical messages provided.

\section{Examples of CDSS in practice}

\subsection{Historical examples: Leeds abdominal pain, MYCIN, HELP, Internist-1}

There have been multiple attempts through history to construct a computer or program, which would assist clinicians with their decisions concerning diagnosis and therapy. Ledley and Lusted published the first article evolving around this idea in 1959. The first really functional CDSS didn't appear until the 1970s. We will review the following historical systems: Leeds abdominal pain, MYCIN, HELP and Internist-1. 
F. T. de Dombal and his co-workers at University of Leeds developed Leeds abdominal pain. It used Bayesian reasoning on basis of surgical and pathological diagnoses. These pieces of information were gathered from thousands of patients and put into systems' database. The Leeds abdominal pain system used sensitivity, specificity and diseaseprevalence data for various signs, symptoms and test results. With help of Bayes' theorem it calculated the probability of seven possible diagnoses resulting in acute abdominal pain: appendicitis, diverticulitis, perforated ulcer, cholecystitis, small-bowel obstruction, pancreatitis, and nonspecific abdominal pain. The system assumed that each patient with abdominal pain had one of these seven conditions, thus selected the most likely diagnose on the basis of recorded observations. Evaluation of the system was done by de Dombal et al. in 1972. It showed that the clinicians' diagnoses were correct in only 65 to 80 percent of the 304 cases, whereas the program's diagnoses were correct in 91.8 percent of cases. Surprisingly, the system has never achieved similar results of diagnostic accuracy in practice outside the Leeds University. The most likely reason for that is the variation of data that clinicians entered into the system for acquiring correct diagnoses (de Dombal, Leaper et al. 1972).

MYCIN was a consultation system that emphasized appropriate management of patients who had infections rather than just finding their diagnosis. The developers of this system formed production rules (IF-THEN rules), on basis of current knowledge about infectious diseases. The MYCIN program determined which rules to use and how to chain them together in order to make decisions about a specific case. System developers could update the system's knowledge structure rapidly by removing, altering, or adding rules, without reprogramming or restructuring other parts of the system (Shortliffe 1976).

The HELP system is actually an integrated hospital information system with the ability to generate alerts when data abnormalities in the patient record are noted. It can output data either automatically, in form of printed reports, or it can display specific information, if so requested. Furthermore, the system has an event-driven mechanism for generation of specialized warnings, alerts and reports (Burke, Classen et al. 1991).

Internist-I was an experimental CDSS designed by Pople and Myers at the University of Pittsburg in 1974. It was a rule-based expert system capable of making multiple, complex diagnoses in internal medicine based on patient observations. The Internist-I was using a tree-structured database that linked symptoms with diseases. The evaluation of the system revealed that it was not sufficiently reliable for clinical application. Nevertheless, the most valuable product of the system was its medical knowledge base. This was used as a basis for successor systems including CADUCEUS and Quick Medical Reference (QMR), a commercialized diagnostic CDSS for internists (Miller, Pople et al. 1982).

\subsection{Selected contemporary examples of CDSS}

\section{ATHENA}

The Athena decision support system was deployed in 2002 as a tool to implement guidelines for hypertension. It encourages blood pressure control and issues recommendations about a suitable choice of therapy, concordant with latest guidelines. It also considers co-morbidities of the specific patient in question. ATHENA DSS has an easily changeable knowledge base that specifies criteria for eligibility, risk stratification, set blood pressure margins, it includes relevant co-morbid states and guideline-recommendation, specific for patients with present co-morbidities. The knowledge base also comprises of preferences for certain drugs within antihypertensive drug groups according to the latest evidence. New pieces of evidence are 
constantly changing protocols of best hypertension management; ATHENA is thus designed to be accessible to clinicians for knowledge base-customization and to custom local interpretations of guidelines according to the local population structure and other factors. The system was designed to be independently integrated into a variety of EMR-systems, and is thus interchangeable and adaptable for various health information-systems. The effectiveness, accuracy and success of implementation has been researched and reviewed on many occasions (Goldstein, Coleman et al. 2004; Lai, Goldstein et al. 2004). Such systems as ATHENA can help with supporting general efforts of national and worldwide health care organizations in effective implementation and stricter following of issued guidelines in various fields of medicine.

In 2006 ATHENA DSS for opioid therapy was developed according to specific guidelines for the management of opioid therapy for chronic pain (Trafton, Martins et al. 2010).

This system uses EON architecture from the Stanford University. EON is a knowledgebased system that aids physicians with care of patients treated according to protocols and guidelines. It is constructed from set of software components that are embedded into a wider health information system, which uses patient-specific data. These software components are designed in such a way to be compiled together in various ways, mixed to create different decision-support functionalities. There are several components that are interrelated:

- Knowledge base encodes descriptions of clinical protocols; it is a source of evidencebased data for all other software components of EON.

- Problem solvers: They determine the right protocol for the patient, i.e. solve a task of placing the patient within the right therapy protocol, by addressing the knowledge base and incorporating patient's attributes (e.g. blood pressure measures and comorbidities).

- Eligibility-determination component addresses the same knowledge base to match the factors in a patient with a certain protocol in order to ascertain if he/she is eligible for such a treatment,

- Database mediator is a conductor between all the components in EON and the database that stores patients' information. More sophisticated mediators already extract the relevant data and make relevant connections before presenting it to an EON problemsolver (Nguyen, Shahar et al. 1997).

\section{ISABEL}

Isabel is a web-based diagnosis decision support system that was created in 2001 by physicians. It offers diagnosis decision support at the point of care. The system is eligible for all aged patients, from neonates to geriatrics. Its database covers major specialties like Internal Medicine, Surgery, Gynecology \& Obstetrics, Pediatrics, Geriatrics, Oncology, Toxicology and Bioterrorism. Isabel produces an instant list of likely diagnoses for a given set of clinical features (symptoms, signs, results of tests and investigations etc), followed by suggesting the administration of suitable drugs. This is executed by reconciling (i.e. patternmatching technology) patient data sets with data sets as described in established medical literature. The system allows clinicians to follow their assumptions about differential diagnoses; it hence restricts searches to specific body systems, relatively to diagnoses in question. The system is interfaced with EMR, which allows it to extract existing diagnoses and other patient-specific data. Furthermore it contains a feature to help clinicians answer their questions with up to date knowledge from textbooks and journals. 
Isabel uses Autonomy's natural language processing software as a search tool. The pattern of the clinical features entered is concept-matched with kernels of knowledge; then the bestmatched kernels of knowledge (diagnoses) are returned for consideration. Autonomy's technology is based on advanced pattern-matching techniques (non-linear adaptive digital signal processing) rooted in the theories of Bayesian Inference and Claude Shannon's Principles of Information (Autonomy 2009). These enable identification of patterns that naturally occur in text, based on the usage and frequency of words or terms that correspond to specific concepts. Based on the predominance of one pattern over another within a piece of information, this technology enables computers to recognize when a particular document in question is about the searched subject. In this way, it extracts a document's digital essence and enables various operations to be automatically performed on that text.

Isabel has been extensively validated and been shown to enhance clinician's cognitive skills and thereby improves patient safety and the quality of patient care (Ramnarayan, Tomlinson et al. 2004; OpenClinical 2006).

\section{LISA}

LISA is a CDSS that consists of two main components. The first is a centralized Oracle database, holding all patient information about drug schedules, blood and toxicity results, doses prescribed etc. The database is accessible by health professionals from different sectors and locations. The second component represents a web-based decision support module, which is using the PROforma guideline development technology to provide advice about dose adjustments in treatment of acute childhood lymphoblastic leukemia. LISA is primarily concerned with providing support in the maintenance illness-period during which drug dose decisions have to be continually monitored and adjusted, as responses to these drugs vary significantly from patient to patient. Decision support is considered useful in this period, since many dose-errors have occurred in practice (Hurt, Fox et al. 2003). In order to give the patient an appropriate dosage of chemotherapeutics, the system uses PROforma decision-making capabilities. It also contains an option, which allows a clinician to prescribe an alternative, not defined in the protocol. PROforma bases its dose adjustment recommendations on five main data inputs defined in the protocol: current state, current platelet and absolute neutrophil count of the blood result on which the decision is being based, number of weeks that the patient has been at the current state and number of weeks that the patient has tolerated treatment.

Evaluational studies of LISA have concluded that the system is likely to be accepted by clinicians. Furthermore, the authors are predicting that the usage of LISA could lead to a decrease in non-compliance with the treatment protocol (Bury, Hurt et al. 2004).

\section{Patient specific modeling}

One of the promising novel concepts useful in CDSSs is Patient-Specific Modeling (PSM), which uses individualized computational models of human pathophysiology to model the dynamics of a wide variety of tissues and organs. It is not a CDSS per-se, we could rather think of it as functionality that adds-on and re-invents the process of clinical decisionmaking. Integrated into CDSSs it has a potential to even further improve diagnosis and optimize clinical treatment by predicting outcomes of therapies and surgical interventions. PSM is being pursued in well-funded projects, like the Virtual Physiological Human initiative (Viceconti, Clapworthy et al. 2007) and the International Physiome Project (Bassingthwaighte 2000). 
Major advantage of PSM is that in contrast to regular diagnostic practices, which are based on averaged clinical trials, it provides tailored treatment and optimizes the individual therapy. While the training and experience of the physician prove to be the most valuable when deciding for the best treatment from an available range, this decision-making process often does not take into account all the data potentially available. Decision-making that bases on the results of simulations, which use models derived from patient-specific data, however, sometimes deal with basically immeasurable physical properties of tissues, e.g. tissue stress in the vessel-wall of a distending abdominal aneurysm (Doyle, Callanan et al. 2009). The results of such simulations enable judgment on effectiveness of a range of potential treatments before they are actually administered, preventing the patient from experiencing unneeded or ineffective treatments. We can think of such theoretical predictive data as descriptors that are used to very accurately asses values of outcome measures in a process of clinical decision-making

PSM has been studied and reviewed in modeling of blood vessels, bones, brain, skeletal muscle, heart and in modeling of the behavior of various tumors (Neal and Kerckhoffs 2010).

There are several basic elements, which are common to all fields of PSM in support of clinical decision-making. Data are obtained from the patient's medical record (EMR), e.g. from a virology genotypic assay or an angiography scan. The data is then used to construct a computational model, which is used to perform a complex workflow of simulations of a proposed protocol of treatment. Molecular dynamics simulations of drugs interacting with a range of viral proteins for example, provide results that are then interpreted to assess the efficacy of the treatment in question. This gives the physician an afore unimaginable ability to choose a treatment based on prior knowledge of how will that specific patient respond to it on a molecular-physiologic basis (Sadiq, Mazzeo et al. 2008).

Such patient-specific simulations require access to comprehensive patient data and an appropriate infrastructure for performing very large numbers of complex and demanding simulations. For this manner heterogeneous supercomputing grid technologies are used, which address, in real time, the outcome of many diverse emergency situations, such as the development and impact of hurricanes and earthquakes (Manos, S. et al. 2008). In the biomedical technology, high-performance computation has been used as a research capacity to investigate the interactions in many tissues with regard to biomolecular function and its interference due to disease, and all that in real time. Such real-time large-scale computation, meet the needs of the clinical environment, thus making "ultra patient-specific" clinical decision-support with PSM increasingly achievable.

Despite the attention that PSM has gotten in recent time, the evaluation of its predictive capability has not yet been tested on a large scale and is thus yet to become a standard in clinical care (Neal and Kerckhoffs 2010).

\section{Good practice example - evaluating patient's health using a hierarchical multi-attribute decision model}

With the possibility of employing CDSS in telemedicine, we take a closer look at a computerized model for the evaluation of a patient's health status in nursing care (Šušteršič et al., 2009). Holistic evaluation of a patient's health status is essential for solid decision making regarding the proper patient treatment. Our solution is based on a hierarchical multi-attribute decision model (HMADM) (Turban et al., 2004; Triantaphyllou, 2010). With 
this approach the practical efficiency of the Henderson's theoretical model of basic living activities (BLA) increases (Henderson \& Nite, 1997).

Let us first present our solution from the structural point of view as described in chapter 2 . With regard to the context axes, it deals with not only inpatients and outpatients but also with community nursing. It assists a nurse in practically all phases of the nursing process, including setting nursing diagnoses and nursing interventions. Its role also includes prevention, screening and chronic disease management. Special emphasis is on alerts and reminders.

The knowledge and data source axes are based on the theoretical BLA model. Data are collected directly from the patients or patients' records. The described CDSS is built upon widely used coding schemas such as ICNP and NANDA.

Decision making process is based on a combination of HMADM and expert systems. The knowledge base consists of a tree of criteria and rule based utility functions for every aggregated node in the tree. This way individual patient data are aggregated in an overall assessment of a patient's health status.

Concerning information delivery, the system is request-based with a special emphasis on the explanation of the evaluation results. Thus, the transparency of the nursing process as a workflow is enhanced.

The proposed HMADM can be applied both when analysing patient's health status and when explaining the results of the evaluation. Despite the holistic nature of the BLA approach, these models are not being sufficiently used in clinical practice (Šušteršič et al., 2002; Ozbolt \& Bakken, 2006). A possible reason for limited use is the fact that a large number of parameters are used to describe a patient's health status. This means that it is difficult to gather information on all the parameters. Furthermore, the process of drawing a conclusion becomes very complex. However, the HMADM methodology helps to address these problems. (Potter \& Perry, 2002; Gordon, 2009)

Increasing the operability of the BLA model can be achieved by understanding the model as a HMADM for evaluating patient's health. The model, which has been based on BLA (Šušteršič et al., 1999; Bohanec et al., 2000), is a comprehensive evaluation tool for the nursing care support process. This part of the patient's record becomes hierarchically structured and gains additional transparency, which facilitates the identification of nursing problems, setting of nursing diagnoses and planning of the appropriate nursing care.

Patient's changes in health status on the basis of the defined indicators are monitored at chosen time intervals. In terms of community nursing, where the model was tested, such intervals coincided with health visits or successive sessions using the tele-nursing approach. The model offers a helping hand to nurses as it reduces the possibility of overlooking problems that could otherwise lead to unwanted healthcare events.

Hierarchical multi-attribute decision models are primarily developed for option evaluation: each option, in this case a patient, is described by values of basic attributes and is evaluated according to the model. The final outcome is an overall evaluation for each option (patient). Furthermore, an evaluation result of the option makes it possible to investigate how the result was obtained. It also provides an insight into what specific attributes can be managed to improve the outcome. (Pöyhönen \& Hämäläinen, 2001; Tsoukias, 2008)

The majority of current multi-attribute decision methods are aimed at the development of quantitative decision models (Triantaphyllou, 2010). In such models, all the attributes are continuous and utility functions are typically defined in terms of the attributes' weight, e.g. as a weighted-sum of lower-level attributes. In practice, however, the difficulty of understanding the underlying data behind the numerical results proves to be a common 
problem. The relationships between the attributes are linear, although the nature of the attributes often requires non-linear interdependence. In other words, different weights can be assigned to a single attribute depending on its relative importance. In contrast, Decision Expert (DEX) methodology (Bohanec \& Rajkovič 1990), which is used in our approach, deals with discrete attributes, usually represented by words rather than numbers. The corresponding utility functions are represented by decision rules. Each rule represents one point or several equi-utility points of function values. The utility (aggregation) functions used in the DEX model are therefore not represented by formulae, such as weighted-sum, but are presented as tables of function values, i.e. decision rules (Rajkovič et al., 1988). This way, a HMADM can be built on non-linear discrete utility functions. It can be compared with the relative weights approach, where the weights depend on parameter values. If a parameter value changes, its relative importance (weight) can also be changed if required. By using the DEX interface, expressing and understanding such utility functions as sets of decision rules becomes suitable for being used in practice.

\subsection{Model for patient's health status evaluation}

A HMADM model, based on the BLA model, was developed by an expert team of nurses for the evaluation of patient's health and was tested in two health centres in Slovenia. This model uses indicators, which are measurable BLA criteria for describing a patient's health condition, as basic attributes. In the criteria tree, the BLA are hierarchically organized and, therefore, interconnected. Utility functions are presented as tables of decision rules. In accordance with those rules, the values and definitions of the higher level criteria are defined by the combination of the values and definitions of the lower level criteria, which enter the model as their predecessors. As a result, a comprehensive evaluation of a patient's health is obtained at the top of the criteria tree. The hierarchical structure of a comprehensive evaluation of the BLA is shown in Table 1, which represents a DEX output in tree form, including criteria descriptions. Each attribute is measured on a five-point scale: "problem to a very high degree" (vhd), "problem to a high degree" (hd), "problem to some degree" (sd), "problem to a lesser degree" (ld) and "no problem" (no).
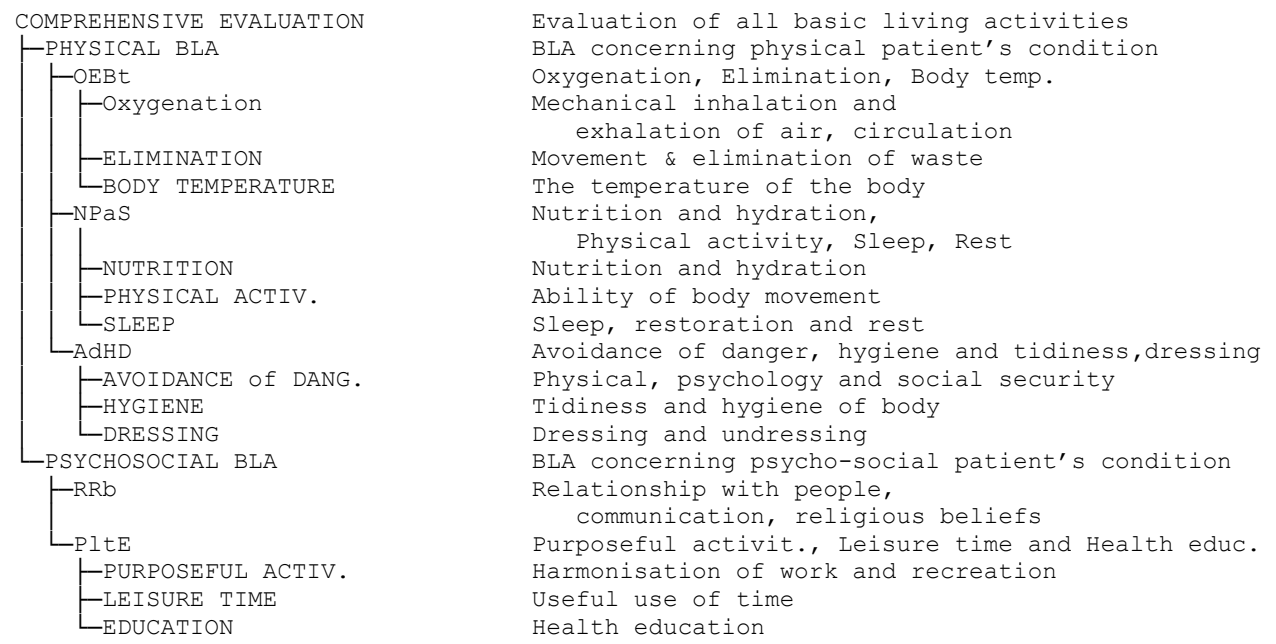

Table 1. DEX printout of hierarchical structure of a comprehensive evaluation of BLA 
The comprehensive evaluation model has 20 attributes comprising of 13 basic and 7 aggregate attributes. These correspond to the main attributes of the BLA criteria, covering items relating both to the patient's physical and psychosocial condition. They include: oxygenation, elimination, body temperature (OEBt); nutrition and hydration, physical activity, sleep and rest (NPaS); avoidance of danger from the environment, hygiene and tidiness, dressing (AdHD); relationships with people, communication, psychological and social needs, including religious beliefs ( $R R b)$; and purposeful activity, leisure time, health education (PLtE). The model also includes seven utility functions, one for each aggregate criterion, including the root of the tree which represents the final evaluation result. Each utility function is defined by a table of decision rules that determine the value of each aggregate attribute on the basis of the mutual dependence on lower-level attributes (immediate predecessor).

Here is an example of a decision rule on how the value of aggregate attribute is defined: if OEBt is assessed as a problem 'to some degree', NPaS as a problem 'to some degree' or worse and AdHD as being on the boundary between 'to a high degree' and 'to some degree', the overall patient's evaluation of physical BLA is evaluated as 'to a high degree'.

The utility functions (sets of decision rules) must be semantically appropriate, i.e. they must express correct nursing knowledge, so verification and validation play important roles. Two steps were included in the present model to achieve this: in the first, a group of chosen experts agreed on utility functions, taking into account existing knowledge from the literature and their experiences and, in the second, nurses validated the knowledge by using decision rules during their practical work.

\subsection{Results of testing in practice}

Testing of the HMADM took place in the community health care environment. The model was used during the entire treatment with a special emphasis on home visits. There were two main objectives of the testing: (i) to validate the nursing knowledge embedded in the model, especially with regard to the decision rules; and (ii) to test the actual operability of the model in practice, i.e. by testing during home visits.

During practical use of the model, transparent decision rules encouraged nurses to critically assess and to validate the nursing knowledge expressed by the rules. Nurses provided comments regarding the appropriateness of the proposed decision rules and the results of the evaluation of the BLA model. The extent to which the HMADM encouraged assessment of the interdependencies between various criteria was also observed.

Testing of the model was monitored during successive home visits. Each nurse used the model to evaluate their patients' health status on regular daily visits. Each nurse carried out between five and seven home visits daily, which were of a preventive and curative nature. At each visit, special emphasis was placed on analysing the patient's health with the set of BLA indicators.

Different patient groups were included, ranging from newborns to the elderly, either due to health promotion, prevention or recovery. Observed were different health problems such as nutrition problems, chronic diseases, wound management etc. An example of a 'comprehensive evaluation of BLA' of a newborn baby (NB) is presented in Table 2, where evaluations from three successive visits (NB1, NB2 and NB3) are listed. The root of the tree, which represents the final overall evaluation, shows how the baby's health condition or their need for nursing care changed from one visit to another. Considerable improvement of the baby's health can be observed. On the first visit the 'Comprehensive evaluation' was 
regarded as a problem to a 'very high degree', on the second visit as a problem to a 'high degree' and, on the third visit, just as a problem to 'some degree'. Table 2 breaks down the comprehensive evaluation result and shows that, at the first visit (NB1), there was a problem to a 'very high degree' for hygiene and education, and a problem to 'some degree' with elimination, sleep, avoidance of danger, $\mathrm{RRb}$ and leisure time. A nurse can easily see all of the existing problems and their actual values (degrees of problem) together with their propagation through the tree of attributes, including interrelationships, up to their influence on the overall evaluation. Such transparency improves understanding of the reasons for changes in the patient's health status and encourages nurses' critical thinking in terms of further actions that they need to take.

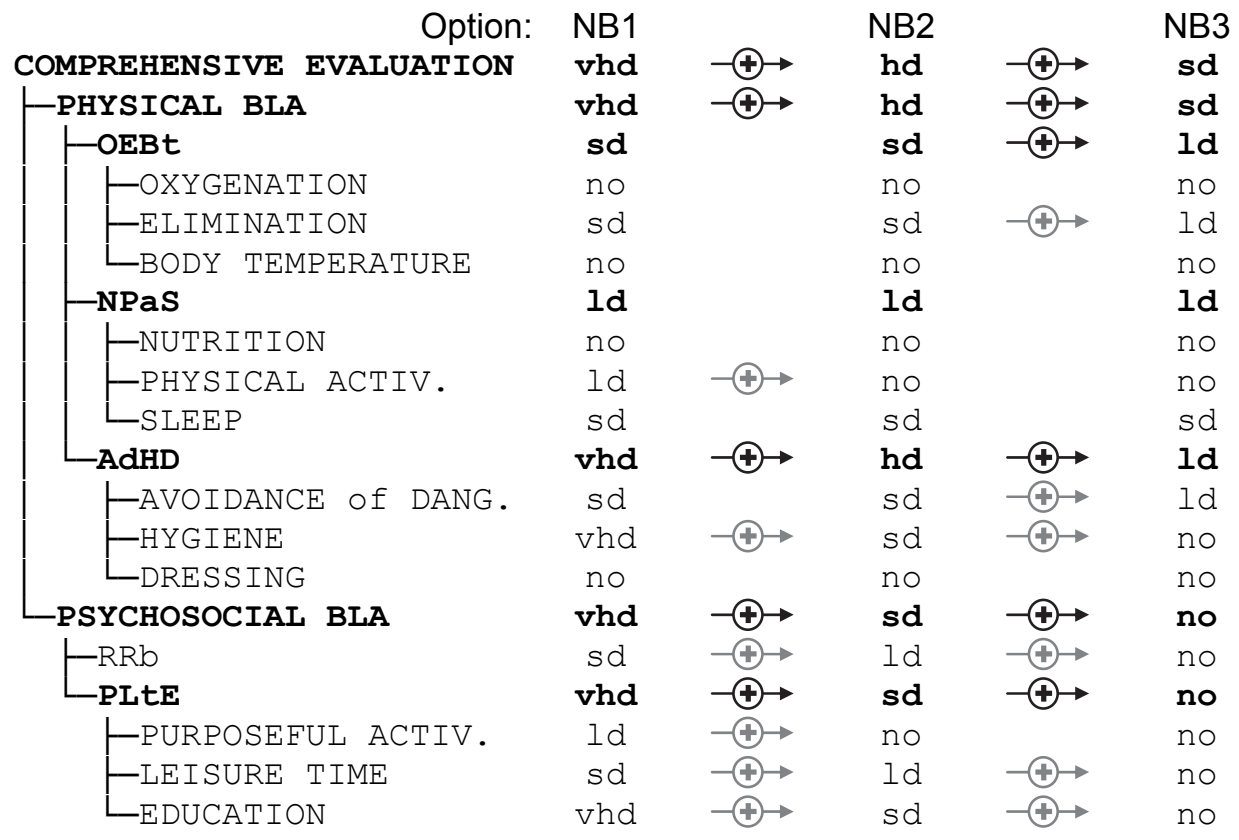

Table 2. Evaluation of a newborn from three successive evaluations

Changes in health indicators can also be followed graphically and Fig. 1 presents the changes during the three visits in the evaluation of physical BLA overall and its four improved sub-attributes: elimination, physical activity, avoidance of danger and hygiene; the condition remained unchanged for sleep. The nurses themselves could choose the indicators, which were then presented as a time series and this customization of the data eliminated the need for further data analysis. Changes in the values of the indicators from one visit to another can also be viewed as a helpful tool in the quality assurance processes.

Nurses who took part in the testing prepared their reports and structured interviews were carried out with each nurse. The reports and interviews served as the basis for a strengths, weaknesses, opportunities and threats (SWOT) analysis. The nurses indentified three main strengths: (i) the model supports holistic understanding necessary for identification of the nature and level of nursing problems; (ii) the computerized hierarchical structure enriches nursing documentation; and (iii) the possibilities of overlooking something important are 
reduced. Among the weaknesses, they emphasized the increased amount of work that was involved in using the model because of the need for consistently following a methodical work process. Opportunities suggested by the nurses were the development of electronic documentation and the consistent holistic evaluation of a patient's health status in the framework of the nurse's competencies. It could contribute to improved patient safety and also to the safety of members of the health team. On the basis of indicators and their values some adverse events can be predicted. If an adverse event occurs, we can explain the reasons and help determine personal responsibility. As the main threat, they listed their apprehension about computer-supported models being perceived as replacing nurses' creative thinking and work.

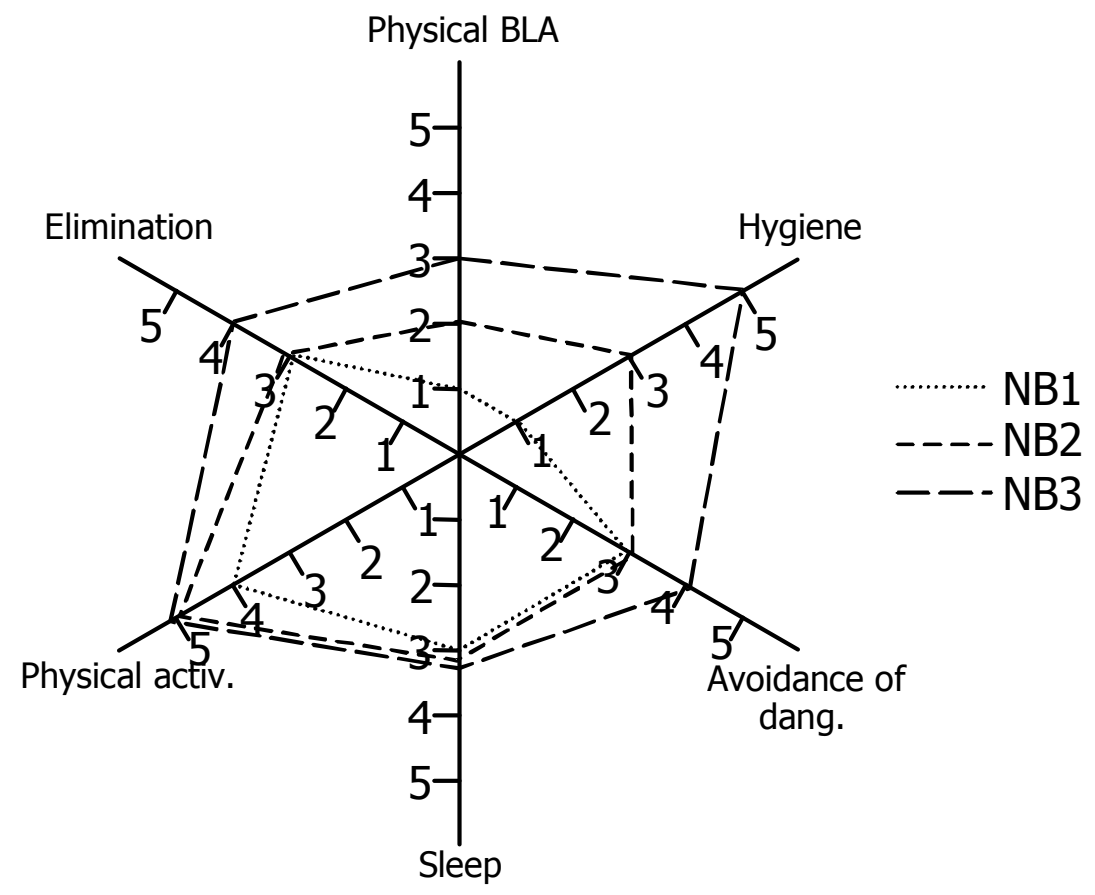

Fig. 1. Transcript of changes of the selected criteria of a newborn's Physical BLA during three visits

\subsection{Towards higher quality of nursing and increased patient safety}

The proposed CDSS uses the BLA model for assessing patients' health. It is built on multiattribute decision-making theory in order to assess a patient's observed condition. The outcome is a computer-calculated comprehensive evaluation based on the model. During clinical testing of the model in practice, transparency appeared to increase along the whole process and not only, as it was initially expected, during the phase of assessing patient health. This implies that patient data can be followed upward through the tree of criteria to the final estimate and an explanation can be provided of how the estimate was obtained and from where it originated. This way a contribution is made to an efficient evaluation that 
provides a holistic and transparent overview of the patient's condition. The structured approach based on the BLA tree assists nurses in keeping up with a large data set and their inter-relationships. This is especially vital when relatively small changes in some indicators may in combination with other indicators result in serious problems for the patient. A better understanding of the outcomes reduces the possibility of overlooking clinically important information. Together with appropriate nursing documentation, this computerized model can contribute to a higher quality of nursing and increased patient safety, as well as increased safety for health team members.

\section{Conclusions, opportunities and roadblocks}

It is clear that systems supporting clinical decision-making of doctors, nurses and other health-workers have an immense potential to benefit in their performance, provision of quality care and, what we strive for - better patient outcomes. In common sense, one can take better care of patients if one has superb knowledge about the clinical matters in question. For example, it could be said that with more information and knowledge a clinician has a better chance of solving a clinical problem in favor of the patient, the hospital and himself. The problem is that nowadays global knowledge about a topic is often overwhelming for a clinician to process at the point of care or in urgent situations. CDSSs incorporate patient-specific data and an applicable, well-structured and current knowledge base or evidence-based guidelines, thus serving the clinician with enhancing his/her clinical decision-making process. Such support of basic cognitive processes involved in medical thinking to some extent relieves the clinician and provides him with new, better-formed and possibly superior methods to take best care of the ill.

Many characteristics of CDSSs are related to clinical effectiveness, functionality, error prevention, potential for acceptance in the clinical world, system portability, costeffectiveness etc., it is therefore important to fully understand the construction and different modalities of CDSS.

The most successful CDSSs deal with therapy critiquing and consulting and/or drug dosing or prescribing. Latest CDSSs use EMR to provide data for analysis, and they provide the ability to the decision-maker to exert the recommended actions with ease, being completely integrated into the information system. Developers of CDSSs should thus be aware that there are factors beyond software and content that must be taken into consideration. Fundamental issues include the level of system integration into clinical workflow and the relevance and timeliness of the clinical messages provided, availability and accessibility of hardware and sufficient technical support and training in use of the system. Reasons for difficulties in implementing CDSS into everyday clinical practice come mainly from programmers' insufficient understanding of medical reasoning and decision analyses. Such systems to some extent pose a threat to diminished clinicians' professional autonomy. Above all there is generally still a lack of awareness of long-term benefits of CDSS-use and lack of desire to change the daily workflow.

To encourage better health-processes, better individual patient care and better population health through CDSSs development, they need to be under constant improvement and their evolvement controlled from an appointed group of experts. To this end the American Medical Informatics Association developed a road map for action on CDSSs, regarding development, implementation and use. This road map comprises of three pillars, with their 
own subsets, which should bring sense into future evolvement and successful implementation of CDSSs (Lyman, Cohn et al. 2010).

1. Best knowledge available when needed;

- $\quad$ Represent clinical knowledge and CDSS interventions in standardized formats.

- $\quad$ There are multiple and most of the time very diverse types of formats used within CDSSs. The patient-specific data and thereafter computerized decision making is usually not exchangeable. This limits free interchange of patients between different institutions on national and international level. It thereby consequently limits the dispersion CDSSs and merits that global usage would bring.

- Collect, organize and distribute clinical knowledge and CDSS interventions in a way that users easily find suitable material and incorporate it in their own information systems.

2. High adoption and effective use

- Address policy, legal and financial barriers

- The health care policy of e.g. European Union should support CDSSs implementation and development. One could expect that as EU directed the electronification of health care it should also direct further evolvement and implementation of CDSSs in everyday clinical practice. Review on research done in this field show multiple beneficial effects of using CDSS, resulting in better patient outcomes, enhanced health-care performance and consequently greater cost effectiveness of electronic health systems.

- With implementing CDSSs in everyday practice, we are obliged to consider the legal consequences of its usage. The obvious conflict is if the decision of CDSS is in discordance with the clinician's opinion. For example, we should consider, what would mean if the clinician wouldn't agree with CDSSs recommendations, which would in common medical sense, be wrong, but the clinicians' actions would anyway result in medical error or even death. How much weight would bear a decision of CDSS? How could we prove that the outcome would be different or better by following CDSS's decision-making? On the other hand CDSSs can impose a regulatory role in following the current medical guidelines. In a way CDSS can prevent a clinician in making medical errors by alerting about e.g. EU-accepted guidelines. CDSS can be viewed as a tool to track clinician's actions. Such information, if comprehensive and detailed, could be of great importance in a court of law.

- Improve the practice of deployment

- Improve the ease of usage

3. Continuous improvement of knowledge and CDSS methods

- Systematically capturing, organizing and examining existing CDSS deployments (e.g. http:/ / www.openclinical.org)

- Advance care-guiding knowledge by using the data readily available in EMR, thus improving clinical knowledge and health management.

- Not only that EMR is time saving and basically essential for health informationsystem (and thus a CDSS) to function successfully and be integrated into a workflow, it is also a source of data for forming new knowledge. EMR can easily 
provide data for clinical research, e.g. statistical analyses of patient outcomes, based on their illnesses and clinician's actions. The results of such analyses could be automatically invested into improving the power of CDSS's decision-making.

Despite all challenges in CDSS development and implementation, these systems have shown to be of great use. Further research and education in this area is therefore essential in order to utilize CDSSs to a higher extent in everyday clinical reality. The implications and positive externalities of CDSSs may well prove to be some of the strongest factors that will influence the health-care development in years to come.

\section{References}

Autonomy, I. (2009). Autonomy Technology Overview. I. Autonomy.

Bassingthwaighte, J. B. (2000). "Strategies for the physiome project." Ann Biomed Eng 28(8): 1043-1058.

Berner, E. S. (2007). Clinical decision support systems : theory and practice. New York, Springer.

Brender, J., E. Ammenwerth, et al. (2006). "Factors influencing success and failure of health informatics systems--a pilot Delphi study.

" Methods Inf Med 45(1): 125-136.

Bohanec, M., Rajkovič, V. (1990). DEX: an expert system shell for decision support. Sistemica, 1, (1990) 145 - 157.

Bohanec, M., Zupan, B., Rajkovič, V. (2000). Applications of qualitative multi-attribute decision models in health care. Int J Med Inform, 58-59, (2000) 191 - 205.

Burke, J. P., D. C. Classen, et al. (1991). "The HELP system and its application to infection control." J Hosp Infect 18 Suppl A: 424-431.

Bury, J., C. Hurt, et al. (2004). "A quantitative and qualitative evaluation of LISA, a decision support system for chemotherapy dosing in childhood acute lymphoblastic leukaemia." Stud Health Technol Inform 107(Pt 1): 197-201.

Coiera, E. (2003). Guide to health informatics. London : New York, NY :, Arnold ; Distributed in the USA by Oxford University Press.

de Dombal, F. T., D. J. Leaper, et al. (1972). "Computer-aided diagnosis of acute abdominal pain." Br Med J 2(5804): 9-13.

Delaney, B. C., D. A. Fitzmaurice, et al. (1999). "Can computerised decision support systems deliver improved quality in primary care?. Interview by Abi Berger." BMJ 319(7220): 1281.

Doyle, B. J., A. Callanan, et al. (2009). "Vessel asymmetry as an additional diagnostic tool in the assessment of abdominal aortic aneurysms." J Vasc Surg 49(2): 443-454.

Garg, A. X., N. K. Adhikari, et al. (2005). "Effects of computerized clinical decision support systems on practitioner performance and patient outcomes: a systematic review." JAMA 293(10): 1223-1238.

General Practice Electronic Decision Support, W. (2000). Strategic future directions / General Practice Electronic Decision Support Workshop. Canberra, A.C.T. :, Dept. of Health and Aged Care.

Goldstein, M. K., R. W. Coleman, et al. (2004). "Translating research into practice: organizational issues in implementing automated decision support for hypertension in three medical centers." J Am Med Inform Assoc 11(5): 368-376. 
Gordon, M. (2009). Manual of Nursing Diagnosis, 12th ed, Jones and Bartlett, ISBN-13: 978-0763771850, Boston

Greenes, R. A. (2007). Clinical decision support : the road ahead. Amsterdam ; Boston, Elsevier Academic Press.

Henderson, V., Nite, G. (1997). Principles and Practice of Nursing, 6th ed, Collier Macmillan, ISBN-10: 0023535806, New York

Holyoak, K. (2005). The Cambridge Handbook of Thinking and Reasoning, Cambridge University Press.

Hurt, C., J. Fox, et al. (2003). Computerised advice on drug dosage decisions in childhood leukaemia: a method and a safety strategy. 9th Conference on Artificial Intelligence in Medicine in Europe, Protaras, Cyprus, Springer.

Kawamoto, K., C. A. Houlihan, et al. (2005). "Improving clinical practice using clinical decision support systems: a systematic review of trials to identify features critical to success." BMJ 330(7494): 765.

Lai, S., M. K. Goldstein, et al. (2004). "Insights from Testing the Accuracy of Recommendations from an Automated Decision Support System for Primary Hypertension: ATHENA DSS." MEDINFO CD: 1706.

Lyman, J. A., W. F. Cohn, et al. (2010). "Clinical decision support: progress and opportunities." J Am Med Inform Assoc 17(5): 487-492.

Manos, S., Z. S., et al. (2008). Life or Death Decision-making: The Medical Case for Largescale, On-demand Grid Computing. CTWatch Quarterly. 4.

Miller, R. A., H. E. Pople, et al. (1982). "Internist-I, an Experimental Computer-Based Diagnostic Consultant for General Internal Medicine." New England Journal of Medicine 307(8): 468-476.

Moxey, A., J. Robertson, et al. (2010). "Computerized clinical decision support for prescribing: provision does not guarantee uptake." J Am Med Inform Assoc 17(1): 25-33.

National Electronic Decision Support, T. (2003). Electronic decision support for Australia's health sector : report to Health Ministers by the National Electronic Decision Support Taskforce. Canberra :, C'wealth Dept. of Health and Ageing.

Neal, M. L. and R. Kerckhoffs (2010). "Current progress in patient-specific modeling." Brief Bioinform 11(1): 111-126.

Nguyen, J. H., Y. Shahar, et al. (1997). "A temporal database mediator for protocol-based decision support." Proc AMIA Annu Fall Symp: 298-302.

OpenClinical, C. (2006). "Isabel - OpenClinical, AI Systems in Clinical Practice." from http://www.openclinical.org/aisp_isabel.html.

Ozbolt, JG., Bakken, S. (2006). Patient-care systems. In: Biomedical Informatics: Computer Applications in Health Care and Biomedicine, 3rd ed. Shortliffe, EH., Cimino, JJ. (Eds.), 564-584, Springer, ISBN-13: 978-0387289861, New York

Patel, V. L., D. R. Kaufman, et al. (2002). "Emerging paradigms of cognition in medical decision-making." J Biomed Inform 35(1): 52-75.

Pearson, S.-A., A. Moxey, et al. (2009). "Do computerised clinical decision support systems for prescribing change practice? A systematic review of the literature (19902007)." BMC Health Services Research 9(1): 154.

Potter, PA., Perry, AG. (2002). Basic Nursing: Essentials for Practice, 5th ed, Mosby, ISBN13: 978-0323016629, St Louis 
Pöyhönen, M., Hämäläinen, RP. (2001). On the convergence of multi-attribute weighting methods. Eur J Oper Res, 129, (2001) 569 - 585.

Rajkovič, V., Bohanec, M., Batagelj, V. (1988). Knowledge engineering techniques for utility identification. Acta Psychol (Amst), 68, (1988) 271 - 286.

Ramnarayan, P., A. Tomlinson, et al. (2004). "A novel diagnostic aid (ISABEL): development and preliminary evaluation of clinical performance." Stud Health Technol Inform 107(Pt 2): 1091-1095.

Remmlinger, E. (2002). Next generation clinical systems: is it time to jump? Annual Healthcare Information and Management Systems Society's Conference. Atlanta, USA. Session 49.

Roberts, M. S. (2011). Decision analysis. Up to date. M. D. Aronson. Waltham, MA, Up to date.

Sadiq, S. K., M. D. Mazzeo, et al. (2008). "Patient-specific simulation as a basis for clinical decision-making." Philos Transact A Math Phys Eng Sci 366(1878): 3199-3219.

Shortliffe, E. H. (1976). Computer-based medical consultations, MYCIN. New York, Elsevier.

Shortliffe, E. H. and J. J. Cimino (2006). Biomedical informatics : computer applications in health care and biomedicine. New York, Springer.

Sim, I. and A. Berlin (2003). "A framework for classifying decision support systems." AMIA Annu Symp Proc: 599-603.

Šušteršič, O., Rajkovič, U., Dinevski, D., Jereb, E., Rajkovič, V. (2009). Evaluating Patients' Health Using a Hierarchical Multi-attribute Decision Model. J Int Med Res, 37, 5, (2009) 1646-1654.

Šušteršič. O., Rajkovič, V., Kljajić, M. (1999). An evaluation of community nursing process in the frame of the international classification of nursing practice. In: ICNP and Telematic Applications for Nurses in Europe - the Telenurse Experience. Mortensen, RA. (Ed.), 243-249, IOS Press, ISBN-13: 978-9051994544,Amsterdam

Šušteršič, O., Rajkovič, V., Leskovar, R., et al (2002). An information system for community nursing. Public Health Nurs, 19, (2002), $184-190$.

Trafton, J. A., S. B. Martins, et al. (2010). "Designing an automated clinical decision support system to match clinical practice guidelines for opioid therapy for chronic pain." Implement Sci 5: 26.

Triantaphyllou, E. (2010). Multi-criteria Decision Making Methods: a Comparative Study, Kluwer Academic Press, ISBN-13: 978-1441948380, Boston

Tsoukiàs, A. (2008). From decision theory to decision aiding methodology. Eur J Oper Res, 187, (2008) $138-161$.

Turban, E., Aronson, J., Liang, TP. (2004). Decision Support Systems and Intelligent Systems, 7th ed, Prentice Hall, ISBN-13: 978-0130461063, New Jersey

Viceconti, M., G. Clapworthy, et al. (2007). Seeding the EuroPhysiome: a roadmap to the virtual physiological human. eHealth, STEP: A strategy for the EuroPhysiome.

Wikipedia, C. (2011). "Inference engine." Retrieved 2 February, 2011, from http://en.wikipedia.org/wiki/Inference_engine.

Wyatt, J. and D. Spiegelhalter (1991). "Field trials of medical decision-aids: potential problems and solutions." Proc Annu Symp Comput Appl Med Care: 3-7. 
Zheng, K., R. Padman, et al. (2005). "Understanding technology adoption in clinical care: clinician adoption behavior of a point-of-care reminder system." Int J Med Inform 74(7-8): 535-543. 


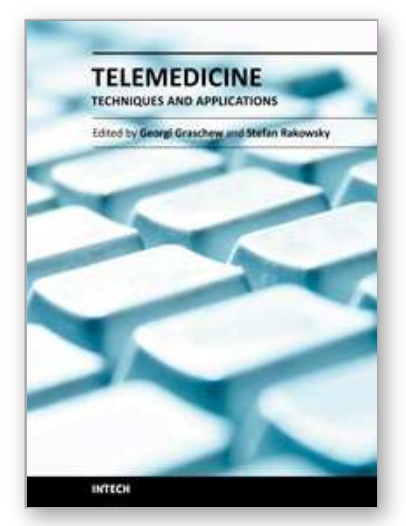

\author{
Telemedicine Techniques and Applications \\ Edited by Prof. Georgi Graschew
}

ISBN 978-953-307-354-5

Hard cover, 514 pages

Publisher InTech

Published online 20, June, 2011

Published in print edition June, 2011

Telemedicine is a rapidly evolving field as new technologies are implemented for example for the development of wireless sensors, quality data transmission. Using the Internet applications such as counseling, clinical consultation support and home care monitoring and management are more and more realized, which improves access to high level medical care in underserved areas. The 23 chapters of this book present manifold examples of telemedicine treating both theoretical and practical foundations and application scenarios.

\title{
How to reference
}

In order to correctly reference this scholarly work, feel free to copy and paste the following:

Dejan Dinevski, Uroš Bele, Tomislav Sarenac, Uroš Rajkovič and Olga Sušteršic (2011). Clinical Decision Support Systems, Telemedicine Techniques and Applications, Prof. Georgi Graschew (Ed.), ISBN: 978-953307-354-5, InTech, Available from: http://www.intechopen.com/books/telemedicine-techniques-andapplications/clinical-decision-support-systems

\section{INTECH}

open science | open minds

\section{InTech Europe}

University Campus STeP Ri

Slavka Krautzeka 83/A

51000 Rijeka, Croatia

Phone: +385 (51) 770447

Fax: +385 (51) 686166

www.intechopen.com

\section{InTech China}

Unit 405, Office Block, Hotel Equatorial Shanghai

No.65, Yan An Road (West), Shanghai, 200040, China

中国上海市延安西路65号上海国际贵都大饭店办公楼 405 单元

Phone: +86-21-62489820

Fax: +86-21-62489821 
(C) 2011 The Author(s). Licensee IntechOpen. This chapter is distributed under the terms of the Creative Commons Attribution-NonCommercialShareAlike-3.0 License, which permits use, distribution and reproduction for non-commercial purposes, provided the original is properly cited and derivative works building on this content are distributed under the same license. 